\title{
Supra Generalized Closed Soft Sets with Respect to an Soft Ideal in Supra Soft Topological Spaces
}

\author{
A. Kandil ${ }^{1, *}$, O. A. E. Tantawy ${ }^{2}$, S. A. El-Sheikh ${ }^{3}$ and A. M. Abd El-latif ${ }^{3}$ \\ ${ }^{1}$ Mathematics Department, Faculty of Science, Helwan University, Helwan, Egypt \\ ${ }^{2}$ Mathematics Department, Faculty of Science, Zagazig University, Zagazig, Egypt \\ ${ }^{3}$ Mathematics Department, Faculty of Education, Ain Shams University, Cairo, Egypt
}

Received: 31 Jul. 2013, Revised: 2 Nov. 2013, Accepted: 3 Nov. 2013

Published online: 1 Jul. 2014

\begin{abstract}
The concept of soft ideal was first introduced by Kandil et al. [13]. In 1999, Molodtsov [22] introduced the concept of soft sets as a general mathematical tool for dealing with uncertain objects. The concept of generalized closed soft sets in soft topological spaces was introduced by Kannan [15] in 2012. The notions of supra soft topological space were first introduced by Kandil et al. [14]. In this paper, we introduce the concept of supra generalized closed soft sets(supra g-closed soft for short) in a supra topological space $(X, \mu, E)$ and study their properties in detail. Also, we introduce the concept of supra generalized closed soft sets with respect to a soft ideal (supra- $\tilde{I} g$-closed soft for short) in a supra topological space $(X, \mu, E)$ and study their properties in detail, which is the extension of the concept of supra generalized closed soft sets.
\end{abstract}

Keywords: Soft sets, Soft topological space,Open soft, Closed soft, Supra soft topological space, Supra open soft, Supra closed soft, Supra g-closed soft, Supra g-open soft, Supra- $\tilde{I} g$-closed soft, Supra- $\tilde{I} g$-open soft sets, soft $I g$-open sets and continuous soft mappings.

\section{Introduction}

The concept of soft sets was first introduced by Molodtsov [22] in 1999 as a general mathematical tool for dealing with uncertain objects. In [22,23], Molodtsov successfully applied the soft theory in several directions, such as smoothness of functions, game theory, operations research, Riemann integration, Perron integration, probability, theory of measurement, and so on.

After presentation of the operations of soft sets [20], the properties and applications of soft set theory have been studied increasingly $[4,16,23,25]$. In recent years, many interesting applications of soft set theory have been expanded by embedding the ideas of fuzzy sets $[1,3,6,18$, $19,20,21,23,24,32]$. To develop soft set theory, the operations of the soft sets are redefined and a uni-int decision making method was constructed by using these new operations [7].
Recently, in 2011, Shabir and Naz [29] initiated the study of soft topological spaces. They defined soft topology on the collection $\tau$ of soft sets over X. Consequently, they defined basic notions of soft topological spaces such as open soft and closed sets, soft subspace, soft closure, soft nbd of a point, soft separation axioms, soft regular spaces and soft normal spaces and established their several properties. Hussain and Ahmad [9] investigated the properties of open (closed) soft, soft nbd and soft closure. They also defined and discussed the properties of soft interior, soft exterior and soft boundary which are fundamental for further research on soft topology and will strengthen the foundations of the theory of soft topological spaces. Kandil et al. [13] introduced the notion of soft ideal in soft set theory. Also, they introduced the notion of soft local function. These concepts are discussed with a view to find new soft

\footnotetext{
*Corresponding author e-mail: Alaa_8560@yahoo.com,dr.Alaa_daby@yahoo.com
} 
topologies from the original one. Kandil et al [14] introduced the notion of supra soft topological spaces, which is wider and more general than the class of soft topological spaces.

In 1970, Levine [18] introduced the notion of $g$-closed sets in topological spaces as a generalization of closed sets.

Indeed ideals are very important tools in general topology. It was the works of Newcomb [26], Rancin [27], Samuels [28] and Hamlet Jankovic [8,10] which motivated the research in applying topological ideals to generalize the most basic properties in general Topology. S. Jafari and $\mathrm{N}$. Rajesh introduced the concept of $g$-closed sets with respect to an ideal which is a extension of the concept of g-closed sets. Recently. K. Kannan [15] introduced the concept of $g$-closed soft sets in a soft topological spaces.

The main purpose of this paper is to introduce the notion of supra generalized closed soft sets(supra g-closed soft for short) in a supra topological space $(X, \mu, E)$ and study their properties in detail. We introduce the concept of supra generalized closed soft sets with respect to a soft ideal (supra- $\tilde{I} g$-closed soft for short) in a supra topological space $(X, \mu, E)$ and study their properties in detail, which is the extension of the concept of supra generalized closed soft sets. Also, we study the relationship between supra- $\tilde{I} g$-closed soft sets, supra- $\tilde{I} g$-open soft sets, supra $g$-closed soft sets and supra $g$-open soft sets. This paper, not only can form the theoretical basis for further applications of topology on soft set, but also lead to the development of information systems.

\section{Preliminaries}

In this section, we present the basic definitions and results of soft set theory which will be needed in the sequel.

Definition 2.1.[22] Let $X$ be an initial universe and $E$ be a set of parameters. Let $P(X)$ denote the power set of $X$ and $A$ be a non-empty subset of $E$. A pair $(F, A)$ denoted by $F_{A}$ is called a soft set over $X$, where $F$ is a mapping given by $F: A \rightarrow P(X)$. In other words, a soft set over $X$ is a parametrized family of subsets of the universe $X$. For a particular $e \in A, F(e)$ may be considered the set of $e$ approximate elements of the soft set $(F, A)$ and if $e \notin A$, then $F(e)=\phi$ i.e

$F_{A}=\{F(e): e \in A \subseteq E, F: A \rightarrow P(X)\}$. The family of all these soft sets denoted by $S S(X)_{A}$.

Definition 2.2.[20] Let $F_{A}, G_{B} \in S S(X)_{E}$. Then $F_{A}$ is soft subset of $G_{B}$, denoted by $F_{A} \tilde{\simeq} G_{B}$, if
(1) $A \subseteq B$, and

(2) $F(e) \subseteq G(e), \forall e \in A$.

In this case, $F_{A}$ is said to be a soft subset of $G_{B}$ and $G_{B}$ is said to be a soft superset of $F_{A}, G_{B} \supseteq F_{A}$.

Definition 2.3.[20] Two soft subset $F_{A}$ and $G_{B}$ over a common universe set $X$ are said to be soft equal if $F_{A}$ is a soft subset of $G_{B}$ and $G_{B}$ is a soft subset of $F_{A}$.

Definition 2.4.[4] The complement of a soft set $(F, A)$, denoted by $(F, A)^{\prime}$, is defined by $(F, A)^{\prime}=\left(F^{\prime}, A\right)$, $F^{\prime}: A \rightarrow P(X)$ is a mapping given by $F^{\prime}(e)=X-F(e)$, $\forall e \in A$ and $F^{\prime}$ is called the soft complement function of $F$.

Clearly $\left(F^{\prime}\right)^{\prime}$ is the same as $F$ and $\left((F, A)^{\prime}\right)^{\prime}=(F, A)$.

Definition 2.5.[29] The difference of two soft sets $(F, E)$ and $(G, E)$ over the common universe $X$, denoted by $(F, E)-(G, E)$ is the soft set $(H, E)$ where for all $e \in E$, $H(e)=F(e)-G(e)$.

Definition 2.6.[29] Let $(F, E)$ be a soft set over $X$ and $x \in$ $X$. We say that $x \in(F, E)$ read as $x$ belongs to the soft set $(F, E)$ whenever $x \in F(e)$ for all $e \in E$.

Definition 2.7.[20] A soft set $(F, A)$ over $X$ is said to be a NULL soft set denoted by $\tilde{\phi}$ or $\phi_{A}$ if for all $e \in A, F(e)=\phi$ (null set).

Definition 2.8. [20] A soft set $(F, A)$ over $X$ is said to be an absolute soft set denoted by $\tilde{A}$ or $X_{A}$ if for all $e \in A, F(e)=$ $X$. Clearly we have $X_{A}^{\prime}=\phi_{A}$ and $\phi_{A}^{\prime}=X_{A}$.

Definition 2.9.[20] The union of two soft sets $(F, A)$ and $(G, B)$ over the common universe $X$ is the soft set $(H, C)$, where $C=A \cup B$ and for all $e \in C$,

$H(e)=\left\{\begin{array}{l}F(e), e \in A-B, \\ G(e), e \in B-A, \\ F(e) \cup G(e), e \in A \cap B .\end{array}\right.$

Definition 2.10.[20] The intersection of two soft sets $(F, A)$ and $(G, B)$ over the common universe $X$ is the soft set $(H, C)$, where $C=A \cap B$ and for all $e \in C$, $H(e)=F(e) \cap G(e)$. Note that, in order to efficiently discuss, we consider only soft sets $(F, E)$ over a universe $X$ in which all the parameter set $E$ are same. We denote the family of these soft sets by $S S(X)_{E}$.

Definition 2.11.[33] Let $I$ be an arbitrary indexed set and $L=\left\{\left(F_{i}, E\right), i \in I\right\}$ be a subfamily of $S S(X)_{E}$.

(1)The union of $L$ is the soft set $(H, E)$, where $H(e)=\bigcup_{i \in I} F_{i}(e)$ for each $e \in E$. We write $\tilde{U}_{i \in I}\left(F_{i}, E\right)=(H, E)$.

(2)The intersection of $L$ is the soft set $(M, E)$, where $M(e)=\bigcap_{i \in I} F_{i}(e)$ for each $e \in E$. We write $\tilde{\bigcap}_{i \in I}\left(F_{i}, E\right)=(M, E)$. 
Definition 2.12.[29] Let $\tau$ be a collection of soft sets over a universe $X$ with a fixed set of parameters $E$, then $\tau \subseteq$ $S S(X)_{E}$ is called a soft topology on $X$ if

(1) $\tilde{X}, \tilde{\phi} \in \tau$, where $\tilde{\phi}(e)=\phi$ and $\tilde{X}(e)=X, \forall e \in E$,

(2)the union of any number of soft sets in $\tau$ belongs to $\tau$,

(3)the intersection of any two soft sets in $\tau$ belongs to $\tau$.

The triplet $(X, \tau, E)$ is called a soft topological space over $X$.

Definition 2.13.[9] Let $(X, \tau, E)$ be a soft topological space. A soft set $(F, A)$ over $X$ is said to be closed soft set in $X$, if its relative complement $(F, A)^{\prime}$ is an open soft set.

Definition 2.14.[9] $\operatorname{Let}(X, \tau, E)$ be a soft topological space. The members of $\tau$ are said to be open soft sets in $X$. We denote the set of all open soft sets over $X$ by $O S(X, \tau, E)$, or $O S(X)$ and the set of all closed soft sets by $C S(X, \tau, E)$, or $C S(X)$.

Definition 2.15.[29] Let $(X, \tau, E)$ be a soft topological space and $(F, E) \in S S(X)_{E}$. The soft closure of $(F, E)$, denoted by $\operatorname{cl}(F, E)$ is the intersection of all closed soft super sets of $(F, E)$ i.e

$\operatorname{cl}(F, E)=\tilde{\cap}\{(H, E):(H, E)$ is closed soft set and $(F, E) \tilde{\subseteq}(H, E)\})$.

Definition 2.16.[33] Let $(X, \tau, E)$ be a soft topological space and $(F, E) \in S S(X)_{E}$. The soft interior of $(G, E)$, denoted by $\operatorname{int}(G, E)$ is the union of all open soft subsets of $(G, E)$ i.e

$\operatorname{int}(G, E)=\tilde{\cup}\{(H, E):(H, E)$ is an open soft set and $(H, E) \tilde{\subseteq}(G, E)\})$.

Definition 2.17.[33] The soft set $(F, E) \in S S(X)_{E}$ is called a soft point in $X_{E}$ if there exist $x \in X$ and $e \in E$ such that $F(e)=\{x\}$ and $F\left(e^{\prime}\right)=\phi$ for each $e^{\prime} \in E-\{e\}$, and the soft point $(F, E)$ is denoted by $x_{e}$.

Definition 2.18.[33] The soft point $x_{e}$ is said to be belonging to the soft set $(G, A)$, denoted by $x_{e} \tilde{\in}(G, A)$, if for the element $e \in A, F(e) \subseteq G(e)$.

Definition 2.19.[33] A soft set $(G, E)$ in a soft topological space $(X, \tau, E)$ is called a soft neighborhood (briefly: nbd) of the soft point $x_{e} \tilde{\in} X_{E}$ if there exists an open soft set $(H, E)$ such that $x_{e} \tilde{\in}(H, E) \tilde{\subseteq}(G, E)$.

A soft set $(G, E)$ in a soft topological space $(X, \tau, E)$ is called a soft neighborhood of the soft $(F, E)$ if there exists an open soft set $(H, E)$ such that $(F, E) \tilde{\in}(H, E) \tilde{\subseteq}(G, E)$. The neighborhood system of a soft point $x_{e}$, denoted by $N_{\tau}\left(x_{e}\right)$, is the family of all its neighborhoods.

Definition 2.20.[29] Let $(X, \tau, E)$ be a soft topological space, $(F, E) \in S S(X)_{E}$ and $Y$ be a non null subset of $X$. Then the sub soft set of $(F, E)$ over $Y$ denoted by $\left(F_{Y}, E\right)$, is defined as follows:

$$
F_{Y}(e)=Y \cap F(e) \forall e \in E .
$$

In other words $\left(F_{Y}, E\right)=\tilde{Y} \tilde{\cap}(F, E)$.

Definition 2.21.[29] Let $(X, \tau, E)$ be a soft topological space and $Y$ be a non null subset of $X$. Then

$$
\tau_{Y}=\left\{\left(F_{Y}, E\right):(F, E) \in \tau\right\}
$$

is said to be the soft relative topology on $Y$ and $\left(Y, \tau_{Y}, E\right)$ is called a soft subspace of $(X, \tau, E)$.

Theorem 2.1.[29] Let $\left(Y, \tau_{Y}, E\right)$ be a soft subspace of a soft topological space $(X, \tau, E)$ and $(F, E) \in S S(X)_{E}$. Then

(1)If $(F, E)$ is an open soft set in $Y$ and $\tilde{Y} \in \tau$, then $(F, E) \in$ $\tau$.

(2) $(F, E)$ is an open soft set in $Y$ if and only if $(F, E)=$ $\tilde{Y} \tilde{\cap}(G, E)$ for some $(G, E) \in \tau$.

(3) $(F, E)$ is a closed soft set in $Y$ if and only if $(F, E)=$ $\tilde{Y} \tilde{\cap}(H, E)$ for some $(H, E)$ is $\tau$-closed soft set.

Definition 2.22.[2] Let $S S(X)_{A}$ and $S S(Y)_{B}$ be families of soft sets on $X$ and $Y$ respectively, $u: X \rightarrow Y$ and $p: A \rightarrow B$ be mappings. Let $f_{p u}: S S(X)_{A} \rightarrow S S(Y)_{B}$ be a mapping. Then;

(1)If $(F, A) \in S S(X)_{A}$. Then the image of $(F, A)$ under $f_{p u}$, written as $f_{p u}(F, A)=\left(f_{p u}(F), p(A)\right)$, is a soft set in $S S(Y)_{B}$ such that

$f_{p u}(F)(b)=$

$\left\{\begin{array}{lc}\cup_{a \in p^{-} 1(b) \cap A} & u(F(a)), \quad p^{-1}(b) \cap A \neq \phi, \\ \phi, & \text { otherwise. }\end{array}\right.$

for all $b \in B$.

(2)If $(G, B) \in S S(Y)_{B}$. Then the inverse image of $(G, B)$ under $f_{p u}$, written as $f_{p u}^{-1}(G, B)=\left(f_{p u}^{-1}(G), p^{-1}(B)\right)$, is a soft set in $S S(X)_{A}$ such that $f_{p u}^{-1}(G)(a)=\left\{\begin{array}{lr}u^{-1}(G(p(a))), \quad p(a) \in B, \\ \phi, \quad \text { otherwise. }\end{array}\right.$ for all $a \in A$.

The soft function $f_{p u}$ is called surjective if $p$ and $u$ are surjective, also is said to be injective if $p$ and $u$ are injective.

Definition 2.23.[11,33] Let $\left(X, \tau_{1}, A\right)$ and $\left(Y, \tau_{2}, B\right)$ be soft topological spaces and $f_{p u}: S S(X)_{A} \rightarrow S S(Y)_{B}$ be a function. Then

(1)The function $f_{p u}$ is called continuous soft (cts-soft) if $f_{p u}^{-1}(G, B) \in \tau_{1} \forall(G, B) \in \tau_{2}$.

(2)The function $f_{p u}$ is called open soft if $f_{p u}(G, A) \in \tau_{2} \forall(G, A) \in \tau_{1}$.

(3)The function $f_{p u}$ is called closed soft if $f_{p u}(G, A) \in \tau_{2}^{\prime} \forall(G, A) \in \tau_{1}^{\prime}$.

(4)The function $f_{p u}$ is called semi open soft if $f_{p u}(G, A) \in$ $\operatorname{SOS}(Y) \forall(G, A) \in \tau_{1}$. 
(5)The function $f_{p u}$ is called semi continuous soft function (semi-cts soft) if $f_{p u}^{-1}(G, B) \in \operatorname{SOS}(X) \forall(G, B) \in \tau_{2}$.

(6)The function $f_{p u}$ is called irresolute soft if $f_{p u}^{-1}(G, B) \in \operatorname{SOS}(X)\left[f_{p u}^{-1}(F, B) \in S C S(X)\right] \forall(G, B) \in$ $\operatorname{SOS}(Y)[(F, B) \in \operatorname{SCS}(Y)]$.

(7)The function $f_{p u}$ is called irresolute open (closed) soft if $f_{p u}(G, A) \in \operatorname{SOS}(Y)\left[f_{p u}(F, A) \in S C S(Y)\right] \forall(G, A) \in$ $\operatorname{SOS}(X)[(F, A) \in \operatorname{SCS}(Y)]$.

Theorem 2.2.[2] Let $S S(X)_{A}$ and $S S(Y)_{B}$ be families of soft sets. For the soft function $f_{p u}: S S(X)_{A} \rightarrow S S(Y)_{B}$, the following statements hold,

(a) $f_{p u}^{-1}\left((G, B)^{\prime}\right)=\left(f_{p u}^{-1}(G, B)\right)^{\prime} \forall(G, B) \in S S(Y)_{B}$.

(b) $f_{p u}\left(f_{p u}^{-1}((G, B))\right) \tilde{\subseteq}(G, B) \forall(G, B) \in S S(Y)_{B}$. If $f_{p u}$ is surjective, then the equality holds.

(c) $(F, A) \tilde{\subseteq} f_{p u}^{-1}\left(f_{p u}((F, A))\right) \forall(F, A) \in S S(X)_{A}$. If $f_{p u}$ is injective, then the equality holds.

(d) $f_{p u}(\tilde{X}) \tilde{\subseteq} \tilde{Y}$. If $f_{p u}$ is surjective, then the equality holds.

(e) $f_{p u}^{-1}(\tilde{Y})=\tilde{X}$ and $f_{p u}\left(\tilde{\phi}_{A}\right)=\tilde{\phi}_{B}$.

(f)If $(F, A) \tilde{\subseteq}(G, A)$, then $f_{p u}(F, A) \simeq f_{p u}(G, A)$.

(g)If

$(F, B) \tilde{\subseteq}(G, B)$,

then

$f_{p u}^{-1}(F, B) \tilde{\simeq} f_{p u}^{-1}(G, B) \forall(F, B),(G, B) \in S S(Y)_{B}$.

(h) $f_{p u}^{-1}[(F, B) \tilde{\cup}(G, B)]=f_{p u}^{-1}(F, B) \tilde{\cup} f_{p u}^{-1}(G, B)$ and

$f_{p u}^{-1}[(F, B) \tilde{\cap}(G, B)]=f_{p u}^{-1}(F, B) \tilde{\cap} f_{p u}^{-1}(G, B)$

$\forall(F, B),(G, B) \in S S(Y)_{B}$.

(I) $f_{p u}[(F, A) \tilde{\cup}(G, A)]=f_{p u}(F, A) \tilde{\cup} f_{p u}(G, A) \quad$ and $f_{p u}[(F, A) \tilde{\cap}(G, A)] \tilde{\subseteq} f_{p u}(F, A) \tilde{\cap} f_{p u}(G, A)$

$\forall(F, A),(G, A) \in S S(X)_{A}$. If $f_{p u}$ is injective, then the equality holds.

Definition 2.24.[13] Let $\tilde{I}$ be a non-null collection of soft sets over a universe $X$ with the same set of parameters $E$. Then $\tilde{I} \subseteq S S(X)_{E}$ is called a soft ideal on $X$ with the same set $E$ if

(1) $(F, E) \in \tilde{I}$ and $(G, E) \in \tilde{I} \Rightarrow(F, E) \tilde{\cup}(G, E) \in \tilde{I}$,

(2) $(F, E) \in \tilde{I}$ and $(G, E) \tilde{\subseteq}(F, E) \Rightarrow(G, E) \in \tilde{I}$,

i.e. $\tilde{I}$ is closed under finite soft unions and soft subsets.

Theorem 2.3.[12] Let $\left(X_{1}, \tau_{1}, A, \tilde{I}\right)$ be a soft topological space with soft ideal, $\left(X_{2}, \tau_{2}, B\right)$ be a soft topological space and $f_{p u}:\left(X_{1}, \tau_{1}, A, \tilde{I}\right) \rightarrow\left(X_{2}, \tau_{2}, B\right)$ be a soft function. Then $f_{p u}(\tilde{I})=\left\{f_{p u}((F, A)):(F, A) \in \tilde{I}\right\}$ is a soft ideal on $X_{2}$.

Definition 2.25. [14] Let $\tau$ be a collection of soft sets over a universe $X$ with a fixed set of parameters $E$, then $\mu \subseteq$ $S S(X)_{E}$ is called a supra soft topology on $X$ with a fixed set $E$ if

(1) $\tilde{X}, \tilde{\phi} \in \mu$,

(2)the union of any number of soft sets in $\mu$ belongs to $\mu$.
The triplet $(X, \mu, E)$ is called a supra soft topological space (or supra soft spaces) over $X$.

Definition 2.26.[14] Let $(X, \mu, E)$ be a supra soft topological space over and $(F, E) \in S S(X)_{E}$. Then the supra soft interior of $(G, E)$, denoted by $\operatorname{int}^{S}(G, E)$ is the soft union of all supra open soft subsets of $(G, E)$. Clearly int $^{S}(G, E)$ is the largest supra open soft set over $X$ which contained in $(G, E)$ i.e $\operatorname{int}^{S}(G, E)=\tilde{\cup}\{(H, E):(H, E)$ is supra open soft set and $(H, E) \tilde{\subseteq}(G, E)\}$.

Definition 2.27.[14] Let $(X, \mu, E)$ be a supra soft topological space over and $(F, E) \in S S(X)_{E}$. Then the supra soft closure of $(F, E)$, denoted by $c l^{s}(F, E)$ is the soft intersection of all supra closed super soft sets of $(F, E)$. Clearly $c^{s}(F, E)$ is the smallest supra closed soft set over $X$ which contains $(F, E)$ i.e $c l^{s}(F, E)=\tilde{\cap}\{(H, E):(H, E)$ is supra closed soft set and $(F, E) \tilde{\subseteq}(H, E)\}$.

\section{Supra generalized closed soft sets}

Kannan [15] introduced generalized closed soft sets in soft topological spaces. In this section we generalize the notions of generalized closed soft sets to supra soft topological spaces.

Definition 3.1. A soft set $(F, E)$ is called a supra generalized closed soft set (supra g-closed soft) in a supra soft topological space $(X, \mu, E)$ if $\operatorname{cl}^{s}(F, E) \tilde{\subseteq}(G, E)$ whenever $(F, E) \underline{\tilde{\subseteq}}(G, E)$ and $(G, E)$ is a supra open soft in $X$.

Example 3.1. Suppose that there are three cars in the universe $X$ given by $X=\left\{h_{1}, h_{2}, h_{3}\right\}$. Let $E=\left\{e_{1}, e_{2}\right\}$ be the set of decision parameters which are stands for "expensive" and "beautiful" respectively.

Let $\left(F_{1}, E\right),\left(F_{2}, E\right)$ be two soft sets over the common universe $\mathrm{X}$, which describe the composition of the cars, where

$F_{1}\left(e_{1}\right)=\left\{h_{2}, h_{3}\right\}, \quad F_{1}\left(e_{2}\right)=\left\{h_{1}, h_{2}\right\}$,

$F_{2}\left(e_{1}\right)=\left\{h_{1}, h_{2}\right\}, \quad F_{2}\left(e_{2}\right)=\left\{h_{1}, h_{3}\right\}$.

Then $\mu=\left\{\tilde{X}, \tilde{\phi},\left(F_{1}, E\right),\left(F_{2}, E\right)\right\}$ is the supra soft topology over $X$. Hence the soft sets $\left(F_{1}, E\right),\left(F_{2}, E\right)$ are supra g-closed soft sets in $(X, \mu, E)$, but the set $(G, E)$ where

$G\left(e_{1}\right)=\left\{h_{2}\right\}, \quad G\left(e_{2}\right)=\left\{h_{1}\right\}$ is not supra g-closed soft in $(X, \mu, E)$.

Remark 3.1. The soft intersection (resp. soft union) of any two supra g-closed soft sets is not supra g-closed soft in general as shown in the following examples.

Examples 3.1. 
(1)In example 3.1, $\left(F_{1}, E\right),\left(F_{2}, E\right)$ are supra g-closed soft in $(X, \mu, E)$, but their soft intersection $\left(F_{1}, E\right) \tilde{\cap}\left(F_{1}, E\right)=(M, E)$ where

$M\left(e_{1}\right)=\left\{h_{2}\right\}, \quad M\left(e_{2}\right)=\left\{h_{1}\right\}$ is not supra g-closed soft.

(2)Suppose that there are four alternatives in the universe of houses $X=\left\{h_{1}, h_{2}, h_{3}, h_{4}\right\}$ and consider $E=\{e\}$ be the single parameter "quality of houses "to be the a linguistic variable. Let $\left(F_{1}, E\right),\left(F_{2}, E\right),\left(F_{3}, E\right)$, $\left(F_{4}, E\right),\left(F_{5}, E\right),\left(F_{6}, E\right),\left(F_{7}, E\right),\left(F_{8}, E\right)$ be eight soft sets over the common universe $\mathrm{X}$ which describe the goodness of the houses, where

$F_{1}(e)=\left\{h_{1}\right\}, \quad F_{2}(e)=\left\{h_{4}\right\}, \quad F_{3}(e)=\left\{h_{1}, h_{4}\right\}$,

$F_{4}(e)=\left\{h_{1}, h_{2}\right\}, \quad F_{5}(e)=\left\{h_{2}, h_{4}\right\}$,

$F_{6}(e)=\left\{h_{1}, h_{2}, h_{3}\right\}, \quad F_{7}(e)=\left\{h_{2}, h_{3}, h_{4}\right\}$,

$F_{8}(e)=\left\{h_{1}, h_{2}, h_{4}\right\}$.

Then $\mu=\left\{\tilde{X}, \tilde{\phi},\left(F_{1}, E\right),\left(F_{2}, E\right),\left(F_{3}, E\right),\left(F_{4}, E\right)\right.$, $\left.\left(F_{5}, E\right),\left(F_{6}, E\right),\left(F_{7}, E\right),\left(F_{8}, E\right)\right\}$ is the supra soft topology over $X$. Hence the sets $\left(F_{1}, E\right),\left(F_{2}, E\right)$ are supra g-closed soft sets in $(X, \mu, E)$, but their soft union $\left(F_{1}, E\right) \tilde{\cup}\left(F_{1}, E\right)=(H, E)$ where

$H(e)=\left\{h_{1}, h_{4}\right\}$ is not supra g-closed soft.

Remark 3.2. Every supra closed soft set is supra g-closed soft. But the converse is not true in general as shown in the following example.

Example 3.2. In example 3.1, $\left(F_{1}, E\right),\left(F_{2}, E\right)$ are supra gclosed soft in $(X, \mu, E)$, but not supra closed soft over $X$.

Theorem 3.1. Let $(X, \mu, E)$ be a supra soft topological space and $(F, E)$ be a supra g-closed soft in $X$. If $(F, E) \tilde{\subseteq}(H, E) \tilde{\subseteq} c l^{s}(F, E)$, then $(H, E)$ is a supra g-closed soft.

Proof. Let $(H, E) \tilde{\subseteq}(G, E)$ and $(G, E) \in \mu$. Since $(F, E) \tilde{\subseteq}(H, E) \tilde{\subseteq}(G, E)$ and $(F, E)$ is supra g-closed soft in $X, \quad$ then $\quad l^{S}(F, E) \tilde{\subseteq}(G, E)$. Hence $c l^{s}(H, E) \tilde{\subseteq} c l^{s}(F, E) \tilde{\subseteq}(G, E)$. Thus $c l^{s}(H, E) \tilde{\subseteq}(G, E)$. Therefore, $(H, E)$ is a supra g-closed soft.

Theorem 3.2. Let $(X, \mu, E)$ be a supra soft topological space. Then $(H, E)$ is supra g-closed soft in $X$ if and only if $c l^{S}(H, E) \backslash(H, E)$ contains only null supra closed soft set.

Proof. Let $(H, E)$ be a supra g-closed soft set, $(F, E)$ be a non null supra closed soft set in $X$ and $(F, E) \tilde{\subseteq} c l^{s}(H, E) \backslash(H, E)$. Then $(F, E)^{\prime}$ is supra open soft, $(F, E) \tilde{\subseteq} c l^{S}(H, E)$ and $(F, E) \tilde{\subseteq}(H, E)^{\prime}$. Hence $(H, E) \tilde{\subseteq}(F, E)^{\prime}$. Since $(H, E)$ is supra g-closed soft. Then $c l^{s}(H, E) \tilde{\subseteq}(F, E)^{\prime}$. Hence $(F, E) \tilde{\subseteq}\left[c l^{s}(H, E)\right]^{\prime}$. This means that $(F, E) \tilde{\subseteq} c l^{s}(H, E) \tilde{\cap}\left[c l^{s}(H, E)\right]^{\prime}=\tilde{\phi}$. Thus $(F, E)=\tilde{\phi}$ which is a contradiction. Therefore, $c l^{s}(H, E) \backslash(H, E)$ contains only null supra closed soft set.
Conversely, assume that $c l^{s}(H, E) \backslash(H, E)$ contains only null supra closed soft set, $(H, E) \tilde{\subseteq}(G, E),(G, E)$ is supra open soft and suppose that $c l^{s}(H, E) \tilde{\Phi}(G, E)$. Then $c l^{s}(H, E) \tilde{\cap}(G, E)^{\prime}$ is a non null supra closed soft subset of $c l^{s}(H, E) \backslash(H, E)$ which is a contradiction. Thus $(H, E)$ is supra g-closed soft in $X$. This completes the proof.

Corollary 3.1. Let $(F, E)$ be supra g-closed soft set. Then $(F, E)$ is supra closed soft if and only if $\mathrm{cl}^{s}(F, E) \backslash(F, E)$ is supra closed soft.

Proof. If $(F, E)$ is supra closed soft, then $c l^{s}(F, E) \backslash(F, E)=\tilde{\phi}$ is supra closed soft. Conversely, suppose that $\mathrm{cl}^{S}(F, E) \backslash(F, E)$ is supra closed soft. Since $(F, E)$ be supra g-closed soft set. Then $c l^{s}(F, E) \backslash(F, E)=\tilde{\phi}$ from Theorem 3.2. Hence $c l^{s}(F, E)=(F, E)$. Thus $(F, E)$ is supra closed soft.

Definition 3.2. A soft set $(F, E)$ is called a supra generalized open soft set (supra g-open soft) in a supra soft topological space $(X, \mu, E)$ if its relative complement $(F, E)^{\prime}$ is supra g-closed soft in $X$.

Theorem 3.3. Let $(X, \mu, E)$ be supra soft topological space. Then the supra soft set $(F, E)$ is supra g-open soft set if and only if $(F, E) \widetilde{\tilde{\subseteq}} i n t^{s}(G, E)$ whenever $(F, E) \tilde{\subseteq}(G, E)$ and $(F, E)$ is supra closed soft in $X$.

Proof. Let $(F, E)$ be a supra g-open soft in $X$, $(F, E) \tilde{\subseteq}(G, E)$ and $(F, E)$ is supra closed soft in $X$. Then $(F, E)^{\prime}$ is supra g-closed soft from Definition 3.2 and $(G, E)^{\prime} \tilde{\subseteq}(F, E)^{\prime}$. Since $(F, E)$ is supra g-open soft in $X$. Then $c l^{s}(G, E)^{\prime} \tilde{\subseteq}(F, E)^{\prime}$. Hence $(F, E) \tilde{\subseteq}\left[c l^{s}(G, E)^{\prime}\right]^{\prime}=i n t^{s}(G, E)$. Conversely, let $(F, E)^{\prime} \tilde{\subseteq}(H, E)$ and $(H, E)$ is supra open soft in $X$. Then $(H, E)^{\prime} \tilde{\subseteq}(F, E)$ and $(H, E)^{\prime}$ is supra closed soft in $X$. Hence $(H, E)^{\prime} \subseteq \operatorname{cint}^{s}(F, E)$ from the necessary condition. Thus $\left[\operatorname{int}^{s}(F, E)\right]^{\prime}=c l^{s}\left[(F, E)^{\prime}\right] \tilde{\subseteq}(H, E)$ and $(H, E)$ is supra open soft in $X$. This means that $(F, E)^{\prime}$ is supra g-closed soft in $X$. Therefore, $(F, E)$ is supra g-open soft set from Definition 3.2. This completes the proof.

Example 3.3. In example $3.1,\left(F_{1}, E\right)^{\prime},\left(F_{2}, E\right)^{\prime}$ are supra g-open soft in $(X, \mu, E)$.

Remark 3.3. Every supra open soft set is supra g-open soft. But the converse is not true in general as shown in the following example.

Example 3.4. In example 3.1, $\left(F_{1}, E\right)^{\prime},\left(F_{2}, E\right)^{\prime}$ are supra g-open soft in $(X, \mu, E)$, but not supra open soft over $X$.

Theorem 3.4. Let $(X, \mu, E)$ be a supra soft topological space and $(F, E)$ be a supra g-open soft in $X$. If $\operatorname{int}^{S}(F, E) \tilde{\subseteq}(H, E) \tilde{\subseteq}(F, E)$, then $(H, E)$ is a supra g-open soft.

Proof. Let $(G, E) \tilde{\subseteq}(H, E)$ and $(G, E) \in \mu$. Since $(G, E) \tilde{\subseteq}(H, E) \tilde{\subseteq}(F, E)$ and $(F, E)$ is supra g-open soft in 
$X$, then $(G, E) \underline{\widetilde{\subseteq}} \operatorname{int}^{S}(F, E)$. Hence $(G, E) \tilde{\widetilde{\subseteq}} i n t^{s}(F, E) \tilde{\widetilde{\subseteq}} i n t^{s}(H, E)$. Thus $(G, E) \tilde{\widetilde{\subseteq}} i n t^{s}(H, E)$. Therefore, $(H, E)$ is a supra g-open soft.

\section{Supra generalized closed soft sets with respect to an soft ideal}

Definition 4.1. A soft set $F_{E} \in S S(X, E)$ is called supra generalized closed soft with respect to a soft ideal $\tilde{I}$ (supra- $\tilde{I} g$-closed soft) in a supra soft topological space $(X, \mu, E)$ if $c l^{s} F_{E} \backslash G_{E} \in \tilde{I}$ whenever $F_{E} \tilde{\subseteq} G_{B}$ and $G_{E} \in \mu$.

Example 4.1. Let $X=\left\{h_{1}, h_{2}, h_{3}\right\}$ be the set of three houses under consideration and $E=\left\{e_{1}, e_{2}\right\}$ be the set of decision parameters which are stands for "wooden" and "green surroundings" respectively.

. Let $\left(F_{1}, E\right),\left(F_{2}, E\right)$ be two soft sets representing the attractiveness of the houses which $\mathrm{Mr} . A$ and $\mathrm{Mr} . B$ are going to buy, where

$F_{1}\left(e_{1}\right)=\left\{h_{2}, h_{3}\right\}, F_{1}\left(e_{2}\right)=\left\{h_{1}, h_{2}\right\}$,

$F_{2}\left(e_{1}\right)=\left\{h_{1}, h_{2}\right\}, F_{2}\left(e_{2}\right)=\left\{h_{1}, h_{3}\right\}$.

Then $\mu=\left\{\tilde{X}, \tilde{\phi},\left(F_{1}, E\right),\left(F_{2}, E\right)\right\}$ is the supra soft topology over $X$. Let $\tilde{I}=\left\{\tilde{\phi},\left(I_{1}, E\right),\left(I_{2}, E\right),\left(I_{3}, E\right)\right\}$ be a soft ideal over $X$, where $\left(I_{1}, E\right),\left(I_{2}, E\right),\left(I_{3}, E\right)$ are soft sets over $X$ defined by

$I_{1}\left(e_{1}\right)=\left\{h_{1}\right\}, F_{1}\left(e_{2}\right)=\phi$,

$I_{2}\left(e_{1}\right)=\phi, I_{2}\left(e_{2}\right)=\left\{h_{3}\right\}$, and

$I_{3}\left(e_{1}\right)=\left\{h_{1}\right\}, I_{3}\left(e_{2}\right)=\left\{h_{3}\right\}$.

So $\left(F_{1}, E\right)$ is a supra- $\tilde{I} g$-closed soft.

Proposition 4.1. Every supra $g$-closed soft set is supra- $\tilde{I} g$ closed soft.

Proof. Let $F_{E}$ be a supra $g$-closed soft set in a supra soft topological space $(X, \mu, E)$ and $F_{E} \tilde{\subseteq} G_{E}$ such that $G_{E} \in \mu$. Since $F_{E}$ is supra $g$-closed soft, then $c l^{s}(F, E) \tilde{\simeq} G_{E}$ and hence $c l^{s}(F, E) \backslash G_{E}=\phi \in \tilde{I}$. Consequently $F_{E}$ is a supra- $\tilde{I} g$-closed soft set.

The following example shows that the converse of the above proposition is not true in general.

Example 4.2. Suppose that there are three cars in the universe $X$ given by $X=\left\{h_{1}, h_{2}, h_{3}\right\}$. Let $E=\left\{e_{1}, e_{2}\right\}$ be the set of decision parameters which are stands for "expensive" and "beautiful" respectively.

Let Let $\left(F_{1}, E\right),\left(F_{2}, E\right),\left(F_{3}, E\right),\left(F_{4}, E\right)$ be four soft sets over the common universe $\mathrm{X}$, which describe the composition of the cars, where

$F_{1}\left(e_{1}\right)=\left\{h_{1}\right\}, F_{1}\left(e_{2}\right)=X$,

$F_{2}\left(e_{1}\right)=\left\{h_{1}, h_{2}\right\}, F_{2}\left(e_{2}\right)=X$,
$F_{3}\left(e_{1}\right)=\left\{h_{3}\right\}, F_{3}\left(e_{2}\right)=X$, and

$F_{4}\left(e_{1}\right)=\left\{h_{1}, h_{3}\right\}, F_{4}\left(e_{2}\right)=X$.

Then $\mu=\left\{\tilde{X}, \tilde{\phi},\left(F_{1}, E\right),\left(F_{2}, E\right),\left(F_{3}, E\right),\left(F_{4}, E\right)\right\}$ is the supra soft topology over $X$. Let $\tilde{I}=\left\{\tilde{\phi},\left(I_{1}, E\right),\left(I_{2}, E\right),\left(I_{3}, E\right)\right\}$ be a soft ideal over $X$, where $\left(I_{1}, E\right),\left(I_{2}, E\right),\left(I_{3}, E\right)$ are soft sets over $X$ defined by

$I_{1}\left(e_{1}\right)=\left\{h_{2}\right\}, F_{1}\left(e_{2}\right)=\phi$,

$I_{2}\left(e_{1}\right)=\left\{h_{3}\right\}, I_{2}\left(e_{2}\right)=\phi$, and

$I_{3}\left(e_{1}\right)=\left\{h_{2}, h_{3}\right\}, I_{3}\left(e_{2}\right)=\phi$.

So $\left(F_{1}, E\right)$ is a supra- $\tilde{I} g$-closed soft but it is not supra g-closed soft.

Theorem 4.1. A supra soft set $(G, E)$ is a supra- $\tilde{I} g$-closed soft set in a supra soft topological space $(X, \mu, E)$ if and only if there exist a supra closed soft set $(F, E)$ such that $(F, E) \underline{\tilde{\subseteq}} c l^{s}(G, E) \backslash(G, E)$ implies $(F, E) \in \tilde{I}$.

Proof. $(\Rightarrow)$ Suppose that $(G, E)$ be a supra-I $I g$-closed soft set and $(F, E)$ be a supra closed soft set such that $(F, E) \tilde{\subseteq} c l^{S}(G, E) \backslash(G, E)$. Then $(G, E) \subseteq(F, E)^{\prime}$. By our assumption, $\quad c l^{S}(G, E) \backslash(F, E)^{\prime} \in \tilde{I}$. But $(F, E) \tilde{\subseteq} c l^{s}(G, E) \tilde{\cap}(F, E)=c l^{s}(G, E) \backslash(F, E)^{\prime}$. Thus $(F, E) \in \tilde{I}$ from Definition 2.24.

$(\Leftarrow)$ Conversely, assume that $(G, E) \tilde{\subseteq}(H, E)$ and $(H, E) \in \mu$. Then $c l^{s}(G, E) \backslash(H, E)=$ $c l^{s}(G, E) \tilde{\cap}(H, E)^{\prime}=c l^{s}(G, E) \tilde{\cap} c l^{s}\left((H, E)^{\prime}\right)$ is a supra closed soft set in $(X, \mu, E)$ and $c l^{s}(G, E) \backslash(H, E) \tilde{\widetilde{C}} c l^{s}(G, E) \backslash(H, E)$. By assumption $c l^{s}(G, E) \backslash(H, E) \in \tilde{I}$. This implies that $(G, E)$ is a supra- $\tilde{I} g$-closed soft.

Theorem 4.2. If $(F, E)$ and $(G, E)$ are supra- $\tilde{I} g$-closed soft sets in a supra soft topological space $(X, \mu, E)$, then $(F, E) \tilde{\cup}(G, E)$ is also supra- $\tilde{I} g$-closed soft in $(X, \mu, E)$.

Proof. Suppose that $F_{E}$ and $G_{E}$ are supra- $\tilde{I} g$-closed soft in $(X, \mu, E)$. Let $F_{E} \tilde{\cup} G_{E} \tilde{\subseteq} H_{E}$ and $H_{E} \in \tau$, then $F_{E} \widetilde{\simeq} H_{E}$ and $G_{E} \tilde{\subseteq} H_{E}$. By assumption $c l^{s} F_{E} \backslash H_{E} \in \tilde{I}$ and $c l^{s} G_{E} \backslash H_{E} \in \tilde{I}$. It follows that $\left[c l^{s} F_{E} \backslash H_{E}\right] \tilde{\cup}\left[c l^{s} G_{E} \backslash H_{E}\right]=c l^{s}\left[\left(F_{E} \tilde{\cup} G_{E}\right] \backslash H_{E} \in \tilde{I}\right.$. Thus $F_{E} \tilde{\cup} G_{E}$ is a supra- $\tilde{I} g$-closed soft.

Theorem 4.3. If $F_{E}$ is supra- $\tilde{I} g$-closed soft in a supra soft topological space $(X, \mu, E)$ and $F_{E} \tilde{\subseteq} G_{E} \tilde{\subseteq} c l^{s} F_{E}$, then $G_{B}$ is supra-II $g$-closed soft in $(X, \mu, E)$.

Proof. Let $F_{E}$ be a supra- $\tilde{I} g$-closed soft, $F_{E} \tilde{\subseteq} G_{E} \tilde{\subseteq} c l^{s} F_{E}$ in $(X, \mu, E)$ and $G_{E} \tilde{\subseteq} H_{E}$ such that $H_{E} \in \mu$. Then $F_{E} \tilde{\subseteq} H_{E}$. Since $F_{E}$ is a supra- $\tilde{I} g$-closed soft, then $c l^{s} F_{E} \backslash H_{E} \in \tilde{I}$. Now, $G_{E} \tilde{\subseteq} c l^{s} F_{E}$ implies that $c l^{s} G_{E} \tilde{\subseteq} c l^{s} F_{E}$. So $c l^{s} G_{E} \backslash H_{E} \tilde{\subseteq} c l^{s} F_{E} \backslash H_{E}$. Thus $c l^{s} G_{E} \backslash H_{E} \in \tilde{I}$. Consequently, $G_{E}$ is a supra- $\tilde{I} g$-closed soft in $(X, \mu, E)$. This completes the proof. 
Remark 4.1. The soft intersection of two supra- $\tilde{I} g$-closed soft sets need not be a supra- $\tilde{I}$-closed soft set as shown in the following example.

Example 4.3. Suppose that there are three dresses in the universe $\mathrm{X}$ given by $X=\left\{h_{1}, h_{2}, h_{3}\right\}$. Let $E=\left\{e_{1}(\right.$ cotton $), e_{2}($ woollen $\left.)\right\}$ be the set of parameters showing the material of the dresses.

Let $\left(F_{1}, E\right),\left(F_{2}, E\right),\left(F_{3}, E\right)$ be three soft sets over the common universe $X$, which describe the composition of the dresses, where

$F_{1}\left(e_{1}\right)=\left\{h_{2}\right\}, F_{1}\left(e_{2}\right)=\left\{h_{1}\right\}$,

$F_{2}\left(e_{1}\right)=\left\{h_{2}\right\}, F_{2}\left(e_{2}\right)=\left\{h_{2}\right\}$, and

$F_{3}\left(e_{1}\right)=\left\{h_{2}\right\}, F_{3}\left(e_{2}\right)=\left\{h_{1}, h_{2}\right\}$.

Then $\mu=\left\{\tilde{X}, \tilde{\phi},\left(F_{1}, E\right),\left(F_{2}, E\right),\left(F_{3}, E\right)\right\}$ is the supra soft topology over $X$. Let $\tilde{I}=\left\{\tilde{\phi},\left(I_{1}, E\right),\left(I_{2}, E\right),\left(I_{3}, E\right)\right\}$ be a soft ideal over $X$, where $\left(I_{1}, E\right),\left(I_{2}, E\right),\left(I_{3}, E\right)$ are soft sets over $X$ defined by

$I_{1}\left(e_{1}\right)=\left\{h_{1}\right\}, F_{1}\left(e_{2}\right)=\phi$,

$I_{2}\left(e_{1}\right)=\phi, I_{2}\left(e_{2}\right)=\left\{h_{1}\right\}$, and

$I_{3}\left(e_{1}\right)=\left\{h_{1}\right\}, I_{3}\left(e_{2}\right)=\left\{h_{1}\right\}$.

So the soft sets $(G, E),(H, E)$ which defined by

$G\left(e_{1}\right)=\left\{h_{1}, h_{2}\right\}, G\left(e_{2}\right)=\phi$ and $H\left(e_{1}\right)=\left\{h_{2}, h_{3}\right\}$, $H\left(e_{2}\right)=\phi$ are supra- $\tilde{I} g$-closed soft sets. But their soft intersection $(G, E) \tilde{\cap}(H, E)=(K, E)$, where $K\left(e_{1}\right)=\left\{h_{2}\right\}, K\left(e_{2}\right)=\phi$ is not supra- $\tilde{I} g$-closed soft.

Theorem 4.4. If $H_{E}$ is supra- $\tilde{I} g$-closed soft set and $F_{E}$ is a supra closed soft in a supra soft topological space $(X, \mu, E)$. Then $H_{E} \tilde{\cap} F_{E}$ is a supra- $\tilde{I} g$-closed soft in $(X, \mu, E)$.

Proof. Assume that $H_{E} \tilde{\cap} F_{E} \tilde{\subseteq} G_{E}$ and $G_{E} \in \mu$. Then $H_{E} \tilde{\subseteq} G_{E} \tilde{\cup} F_{E}^{\prime}$. Since $H_{E}$ is a supra- $\tilde{I} g$-closed soft set. It follows that $\mathrm{cl}^{s} H_{E} \backslash\left[G_{E} \tilde{\cup} F_{E}^{\prime}\right] \in \tilde{I}$. Now, $c l^{s}\left[H_{E} \tilde{\cap} F_{E}\right] \tilde{\subseteq} c l^{s} H_{E} \tilde{\cap} c l^{s} F_{E}=c l^{s} H_{E} \tilde{\cap} F_{E}=$ $\left[c l^{s} H_{E} \tilde{\cap} F_{E}\right] \backslash F_{E}^{\prime}$. Thus $c l^{s}\left[H_{E} \tilde{\cap} F_{E}\right] \backslash G_{E} \tilde{\subseteq}\left[c l^{s} H_{E} \tilde{\cap} F_{E}\right] \backslash$ $\left[G_{E} \tilde{\cup} F_{E}^{\prime}\right] \tilde{\subseteq} c l^{s} H_{E} \backslash\left[G_{E} \tilde{\cup} F_{E}^{\prime}\right] \in \tilde{I}$. Hence $H_{E} \tilde{\cap} F_{E}$ is a supra- $\tilde{I} g$-closed soft set.

\section{Soft generalized open sets with respect to soft ideal}

Definition 5.1. A soft set $F_{E} \in S S(X, E)$ is called supra generalized open soft set with respect to a soft ideal $\tilde{I}$ (supra- $\tilde{I} g$-open soft) in a supra soft topological space $(X, \mu, E)$ if and only if its relative complement $F_{E}^{\prime}$ is a supra- $\tilde{I} g$-closed soft in $(X, \mu, E)$.

Theorem 5.1. A supra soft set $(F, E)$ is a supra $\tilde{I} g$-open soft set in a supra soft topological space $(X, \mu, E)$ if and only if $G_{E} \backslash I_{E} \tilde{\tilde{\subseteq}} i n t^{s} F_{E}$ for some $I_{E} \in \tilde{I}$, whenever $G_{E} \tilde{\subseteq} F_{E}$ and $G_{E}$ is supra closed soft in $(X, \mu, E)$.
Proof. $(\Rightarrow)$ Suppose that $F_{E}$ is a supra- $\tilde{I} g$-open soft set. Let $G_{E} \tilde{\subseteq} F_{E}$ such that $G_{E}$ is a supra closed soft. We have $F_{E}^{\prime} \tilde{\subseteq} G_{E}^{\prime}, F_{E}^{\prime}$ is a supra- $\tilde{I}$-closed soft and $G_{E}^{\prime} \in \mu$. It follows that $c l^{s} F_{E}^{\prime} \backslash G_{E}^{\prime} \in \tilde{I}$ from Definition 3.1. This implies that $c l^{s} F_{E}^{\prime} \backslash G_{E}^{\prime}=I_{E} \in \tilde{I}$ for some $I_{E} \in \tilde{I}$, and then $c l^{s} F_{E}^{\prime} \backslash G_{E}^{\prime}=c l^{s} F_{E}^{\prime} \tilde{\cap} G_{E}=I_{E} \in \tilde{I}$, so $\left[c l^{s} F_{E}^{\prime} \tilde{\cap} G_{E}\right] \tilde{\cup} G_{E}^{\prime}=I_{E} \tilde{\cup} G_{E}^{\prime}$. This implies that $c l^{s} F_{E}^{\prime} \tilde{\widetilde{G}} c l^{s} F_{E}^{\prime} \tilde{\cup} G_{E}^{\prime}=I_{E} \tilde{\cup} G_{E}^{\prime}$. Hence $c l^{s} F_{E}^{\prime} \tilde{\subseteq} G_{E}^{\prime} \tilde{\cup} I_{E}$ for some $I_{E} \in \tilde{I}$. So $\left(G_{E}^{\prime} \tilde{\cup} I_{E}\right)^{\prime} \tilde{\subseteq}\left[c l^{s} F_{E}^{\prime}\right]^{\prime}=i n t^{s} F_{E}$. Therefore, $G_{E} \backslash I_{E}=G_{E} \tilde{\cap} I_{E}^{\prime} \tilde{\subseteq} i n t^{s} F_{E}$.

$(\Leftarrow)$ Conversely, assume that $F_{E}$ be a supra soft set. We want to prove that $F_{E}$ is a supra- $\tilde{I} g$-open soft set. It is sufficient to prove that $F_{E}^{\prime}$ is a supra- $\tilde{I} g$-closed soft set. So, let $F_{E}^{\prime} \tilde{\subseteq} G_{E}$ such that $G_{E} \in \mu$. Hence $G_{E}^{\prime} \tilde{\subseteq} F_{E}$. By assumption, $G_{E}^{\prime} \backslash I_{E} \tilde{\subseteq} i n t^{s} F_{E}=\left[c l^{s} F_{E}^{\prime}\right]^{\prime}$ for some $I_{E} \in \tilde{I}$. Hence $\quad c^{s} F_{E}^{\prime} \tilde{\subseteq}\left[G_{E}^{\prime} \backslash I_{E}\right]^{\prime}=G_{E} \tilde{\cup} I_{E}$. Thus $c l^{s} F_{E}^{\prime} \backslash G_{E} \tilde{\subseteq}\left[G_{E}^{\prime} \backslash I_{E}\right]^{\prime}=\left[G_{E} \tilde{\cup} I_{E}\right] \backslash G_{E}=\left[G_{E} \tilde{\cup} I_{E}\right] \tilde{\cap} G_{E}^{\prime}=$ $I_{E} \tilde{\cap} G_{E}^{\prime} \tilde{\subseteq} I_{E} \in \tilde{I}$. This shows that $c l^{s} F_{E}^{\prime} \backslash G_{E} \in \tilde{I}$. Therefore, $F_{E}^{\prime}$ is a supra- $\tilde{I} g$-closed soft set and hence $F_{E}$ is a supra- $\tilde{I} g$-open soft set. This completes the proof.

Definition 5.2. Two soft sets $F_{E}$ and $G_{E}$ are said to be supra soft separated sets in a supra soft topological space $(X, \mu, E)$ if $c l^{s} F_{E} \tilde{\cap} G_{E}=\tilde{\phi}$ and $F_{E} \tilde{\cap} c l^{s} G_{E}=\tilde{\phi}$.

Theorem 5.2. If $A_{E}$ and $B_{E}$ are supra soft separated and supra- $\tilde{I} g$-open soft sets in a supra soft topological space $(X, \tau, E)$, then $A_{E} \tilde{\cup} B_{E}$ is a supra- $\tilde{I} g$-open soft in $(X, \mu, E)$.

Proof. Suppose that $A_{E}$ and $B_{E}$ are supra soft separated and supra- $\tilde{I} g$-open soft sets in a supra soft topological space $(X, \tau, E)$ and $F_{E}$ be a supra closed soft set such that $F_{E} \tilde{\subseteq} A_{E} \tilde{\cup} B_{E}$. Then $F_{E} \tilde{\cap} c l^{s} A_{E} \tilde{\subseteq}\left[A_{E} \tilde{\cup} B_{E}\right] \tilde{\cap} c l^{s} A_{E}=A_{E}$ and $F_{E} \tilde{\cap} c l^{s} B_{E} \tilde{\subseteq} B_{E}$ from Definition 5.2. $\left[\left(F_{E} \tilde{\cap} c l^{s} A_{E}\right] \backslash D_{E} \tilde{\subseteq} i n t^{s} A_{E}\right.$ and $\left[F_{E} \tilde{\cap} c l^{s} B_{E}\right] \backslash C_{E} \tilde{\tilde{C}} i n t^{s} B_{E}$ for some $D_{E}, C_{E} \in \tilde{I}$ from Theorem 5.1. This means that $\left[F_{E} \tilde{\cap} c l^{s} A_{E}\right] \backslash i n t^{s} A_{E} \in I$ and $\quad\left(F_{E} \tilde{\cap} c l^{s} B_{E}\right) \backslash$ int $^{s} A_{E} \in I$. Then $\left[\left(F_{E} \tilde{\cap} c l^{s} A_{E}\right) \backslash i n t^{s} A_{E}\right] \tilde{\cup}\left[\left(F_{E} \tilde{\cap} c l^{s} B_{E}\right) \backslash i n t^{s} B_{E}\right] \in \tilde{I}$. Hence $\left[F_{E} \tilde{\cap}\left(c l^{s} A_{E} \tilde{\cup} c l^{s} B_{E}\right)\right] \backslash\left[i n t^{s} A_{E} \tilde{\cup} i n t^{s} B_{E}\right] \in \tilde{I}$. But $F_{E}=F_{E} \tilde{\cap}\left(A_{E} \tilde{\cup} B_{E}\right) \tilde{\subseteq} F_{E} \tilde{\cap}\left[c l^{s}\left(A_{E} \tilde{\cup} B_{E}\right)\right]$, and we have $F_{E} \backslash \quad \operatorname{int}^{s}\left(A_{E} \tilde{\cup} B_{E}\right) \tilde{\subseteq}\left(F_{E} \tilde{\cap}\left(c l^{s}\left[A_{E} \tilde{\cup} B_{E}\right]\right)\right) \backslash$ $\operatorname{int}^{s}\left(A_{E} \tilde{\cup} B_{E}\right) \tilde{\subseteq}\left(F_{E} \tilde{\cap}\left[c l^{s}\left(A_{E} \tilde{\cup} B_{E}\right)\right] \backslash\right.$ int $\left.^{s} A_{E} \tilde{\cup} i n t^{s} B_{E}\right) \in \tilde{I}$. Now, take $G_{E}=F_{E} \backslash \operatorname{int}^{s}\left(A_{E} \tilde{\cup} B_{E}\right) \in \tilde{I}$. Then $F_{E} \backslash G_{E}=F_{E} \backslash\left[F_{E} \backslash i n t^{s}\left(A_{E} \tilde{\cup} B_{E}\right)\right] \tilde{\subseteq} i n t^{s}\left(A_{E} \tilde{\cup} B_{E}\right)$. Hence $F_{E} \backslash G_{E} \tilde{\simeq} i n t^{s}\left(A_{E} \tilde{\cup} B_{E}\right)$ for some $G_{E} \in \tilde{I}$. Therefore, $A_{E} \tilde{\cup} B_{E}$ is a supra- $\tilde{I} g$-open soft in $(X, \mu, E)$ from Theorem 5.1 .

Corollary 5.1. If $A_{E}$ and $B_{E}$ are supra- $\tilde{I} g$-closed soft sets in a supra soft topological space $(X, \mu, E)$ such that $A_{E}^{\prime}$ and $B_{E}^{\prime}$ are supra soft separated sets, then $A_{E} \tilde{\cap} B_{E}$ is a supra- $\tilde{I} g$ closed soft in $(X, \mu, E)$.

Proof. Obvious from Theorem 5.2. 
Theorem 5.3. If $A_{E}$ and $B_{E}$ are supra- $\tilde{I} g$-open soft sets in a supra soft topological space $(X, \mu, E)$, then $A_{E} \tilde{\cap} B_{E}$ is a supra-I $\tilde{I}$-open soft in $(X, \mu, E)$.

Proof. Let $A_{E}$ and $B_{E}$ are supra- $\tilde{I} g$-open soft sets in a supra soft topological space $(X, \mu, E)$, then $A_{E}^{\prime}$ and $B_{E}^{\prime}$ are supra- $\tilde{I} g$-closed soft sets. Hence $\left(A_{E} \tilde{\cap} B_{E}\right)^{\prime}=A_{E}^{\prime} \tilde{\triangle} B_{E}^{\prime}$ is a supra- $\tilde{I} g$-closed soft from Theorem 4.2. Therefore, $A_{E} \tilde{\cup} B_{E}$ is a supra- $\tilde{I} g$-closed soft.

Theorem 5.4. Let $A_{E}$ be a supra- $\tilde{I} g$-open soft in a supra soft topological space $(X, \mu, E)$ such that $\operatorname{int}^{s} A_{E} \tilde{\subseteq} B_{E} \tilde{\subseteq}$ for some $B_{E} \in S S(X)_{E}$. Then $B_{E}$ is a supra- $\tilde{I} g$-open soft in $(X, \mu, E)$.

Proof. Let $A_{E}$ be a supra- $\tilde{I} g$-open soft in a supra soft topological space $(X, \mu, E)$ such that $\operatorname{int}^{s} A_{E} \tilde{\subseteq} B_{E} \tilde{\subseteq}$ for some $B_{E} \in S S(X)_{E}$. Then $A_{E}^{\prime} \tilde{\subseteq} B_{E}^{\prime} \tilde{\subseteq}\left(i n t^{s} A_{E}\right)^{\prime}=c l^{s}\left(A_{E}^{\prime}\right)$ and $A_{E}^{\prime}$ is a supra- $\tilde{I} g$-closed soft. Hence $B_{E}^{\prime}$ is a supra- $\tilde{I} g$-closed soft from Theorem 4.3. Therefore, $B_{E}$ is a supra-II $g$-open soft in $(X, \mu, E)$.

Theorem 5.5. A soft set $A_{E}$ is a supra- $\tilde{I} g$-closed soft in a supra soft topological space $(X, \mu, E)$ if and only if $c l^{s} A_{E} \backslash$ $A_{E}$ is a supra- $\tilde{I} g$-open soft.

Proof. $(\Rightarrow)$ Let $F_{E} \tilde{\subseteq} c l^{s} A_{E} \backslash A_{E}$ and $F_{E}$ is a supra closed soft set. Then $F_{E} \in \tilde{I}$ from Theorem 4.1. Hence, there exists $I_{E} \in \tilde{I}$ such that $F_{E} \backslash I_{E}=\tilde{\phi}$. Thus $F_{E} \backslash I_{E}=\tilde{\phi} \tilde{\subseteq} \operatorname{int}^{s}\left[c l^{s} A_{E} \backslash A_{E}\right]$. Therefore, $c l^{s} A_{E} \backslash A_{E}$ is a supra- $\tilde{I} g$-open soft from Theorem 5.1.

$(\Leftarrow) \quad$ Let $A_{E} \tilde{\subseteq} G_{E}$ such that $G_{E} \in \mu$. Then $c l^{s} A_{E} \tilde{\cap} G_{E}^{\prime} \tilde{\subseteq} c l^{s} A_{E} \tilde{\cap} A_{E}^{\prime}=c l^{s} A_{E} \backslash A_{E}$. By hypothesis, $\left[c l^{s} A_{E} \tilde{\cap} G_{E}^{\prime}\right] \backslash I_{E} \tilde{\subseteq} i n t^{s}\left[c l^{s} A_{E} \backslash A_{E}\right]=\tilde{\phi}$, for some $I_{E} \in \tilde{I}$ from Theorem 5.1. This implies that $c l^{s} A_{E} \tilde{\cap} G_{E}^{\prime} \tilde{\subseteq} I_{E} \in \tilde{I}$. Therefore, $c l^{s} A_{E} \backslash G_{E} \in \tilde{I}$. Thus $A_{E}$ is a supra- $\tilde{I} g$-closed soft.

Theorem 5.6. Let $\left(X_{1}, \mu_{1}, A\right),\left(X_{2}, \mu_{2}, B\right)$ be supra soft topological spaces. Let $f_{p u}: S S\left(X_{1}\right)_{A} \rightarrow S S\left(X_{2}\right)_{B}$ be closed and continuous soft function. If $A_{E} \in S S(X, E)$ is a supra- $\tilde{I} g$-closed soft in $\left(X, \mu_{1}, A\right)$, then $f_{p u}\left(A_{E}\right)$ is a supra- $f_{p u}(\tilde{I}) g$-closed soft in $\left(X_{2}, \mu_{2}, B\right)$, where $f_{p u}(\tilde{I})=\left\{f_{p u}\left(I_{E}\right): I_{E} \in \tilde{I}\right\}$.

Proof. Let $A_{E} \in S S(X)_{A}$ be a supra- $\tilde{I}$-closed soft in $\left(X, \mu_{1}, A\right)$ and $f_{p u}\left(A_{E}\right) \tilde{\subseteq} G_{E}$ for some $G_{E} \in \mu_{2}$. Then $A_{E} \tilde{\subseteq} f_{p u}^{-1}\left(G_{E}\right)$. It follow that $c l^{s} A_{E} \backslash f_{p u}^{-1}\left(G_{E}\right) \in \tilde{I}$ from Definition 3.1. Hence $f_{p u}\left(c l^{s} A_{E}\right) \backslash G_{E} \in f_{p u}(\tilde{I})$ from Theorem 2.2. Since $f_{p u}$ is a closed soft function, then $f_{p u} c l^{s}\left(A_{E}\right)$ is a supra closed soft in $\mu_{2}$ from Definition 2.23. Thus $c l^{s}\left(f_{p u}\left(A_{E}\right)\right) \tilde{\subseteq} c l^{s}\left[f_{p u} c l^{s}\left(A_{E}\right)\right]=f_{p u} c l^{s}\left(A_{E}\right)$. This implies that $c l^{s}\left(f_{p u}\left(A_{E}\right)\right) \backslash G_{E} \tilde{\subseteq} f_{p u}\left(c l^{s} A_{E}\right) \backslash G_{E} \in f_{p u}(\tilde{I})$. Therefore, $f_{p u}\left(A_{E}\right)$ is a supra- $f_{p u}(I) g$-closed soft in $\left(X_{2}, \mu_{2}, B\right)$. This completes the proof.

\section{Conclusion}

Topology is an important and major area of mathematics and it can give many relationships between other scientific areas and mathematical models. Recently, many scientists have studied the soft set theory, which is initiated by Molodtsov and easily applied to many problems having uncertainties from social life. In the present work, we have continued to study the properties of soft topological spaces. We introduce the notions of supra g-closed soft, supra g-open sets, supra-I $\tilde{I}$-closed soft sets, supra-II $g$-open soft sets and have established several interesting properties. Because there exists compact connections between soft sets and information systems [25,30], we can use the results deducted from the studies on soft topological space to improve these kinds of connections. We see that this paper will help researcher enhance and promote the further study on soft topology to carry out a general framework for their applications in practical life.

Granular computing is a recent approach in the field of computer science that uses topological structure as granulation models. The suggested approach for supra-I $\tilde{g}$-closed soft sets give new methods for generating the classes of subsets whose lower and upper approximations are contained in elementary sets which in turn help in the process of decision making under both quantities and qualitative information.

\section{Acknowledgements}

The authors express their sincere thanks to the reviewers for their careful checking of the details and for helpful comments that improved this paper. The authors are also thankful to the editors-in-chief and managing editors for their important comments which helped to improve the presentation of the paper.

\section{References}

[1] B. Ahmad and A. Kharal, On fuzzy soft sets, Advances in Fuzzy Systems, 1-6 (2009).

[2] B. Ahmad and A. Kharal, Mappings of soft classes, to appear in New Math. Nat. Comput.

[3] H. Aktas and N. agman, Soft sets and soft groups, Information Sciences, 1, 2726-2735 (2007).

[4] M. I. Ali, F. Feng, X. Liu, W. K. Min and M. Shabir, On some new operations in soft set theory, Computers and Mathematics with Applications, 57, 1547-1553 (2009). 
[5] A. Aygnoglu and H. Aygn, Introduction to fuzzy soft groups, Computers and Mathematics with Applications, 58, 12791286 (2009).

[6] N. agman and F.itak, S. Enginoglu, Fuzzy parameterized fuzzy soft set theory and its applications, Turkish Journal of Fuzzy Systems, 1, 21-35 (2010).

[7] N.agman and S. Enginoglu, Soft set theory and uniint decision making, European Journal of Operational Research, 207, 848-855 (2010).

[8] T. R. Hamlett and D. Jankovic, Compatible extensions of ideals, Boll. Un. Mat. Ita., 7, 453-456 (1992).

[9] S. Hussain and B. Ahmad, Some properties of soft topological spaces, Comput. Math. Appl., 62, 4058-4067 (2011).

[10] D. Jankovic, T. R. Hamlett, New topologies from old via ideals, Amer. Math. Month., 97, 295-310 (1990).

[11] A.Kandil, O. A. E. Tantawy, S. A. El-Sheikh and A. M. Abd El-latif, $\gamma$-operation and decompositions of some forms of soft continuity in soft topological spaces. To appear in the journal Annals of Fuzzy Mathematics and Informatics (AFMI).

[12] A.Kandil, O. A. E. Tantawy, S. A. El-Sheikh and A. M. Abd El-latif, Semi-soft compactness via soft ideals. Submitted for publication.

[13] E. Catmull, J. Clark, Recursively generated B-spline surfaces on arbitrary topological meshes, Computer-Aided Design, 10, 350-355 (1978).

[14] MAX J. EGENHOFER \& ROBERT D. FRANZOSA, Pointset topological spatial relations, International Journal of Geographical Information Systems, 5, 161-174 (1991).

[15] K. Kannan, Soft generalized closed sets in soft topological spaces, Journal of Theoretical and Applied Technology, 37, 17-21 (2012).

[16] D. V. Kovkov, V. M. Kolbanov and D. A. Molodtsov, Soft sets theory-based optimization, Journal of Computer and Systems Sciences International, 46, 872-880 (2007).

[17] N. Levine, Generalized closed sets in topology, Rend. Circ. Mat. Palermo, 19, 89-96 (1970).

[18] P. K. Maji, R. Biswas and A. R. Roy, Fuzzy soft sets, Journal of Fuzzy Mathematics, 9, 589-602 (2001).

[19] P. K. Maji, R. Biswas and A. R. Roy, Intuitionistic fuzzy soft sets, Journal of Fuzzy Mathematics, 9, 677-691 (2001).

[20] P. K. Maji,R. Biswas and A. R. Roy, Soft set theory, Computers and Mathematics with Applications, 45, 555-562 (2003)

[21] P. Majumdar, and S. K. Samanta, Generalised fuzzy soft sets, Computers and Mathematics with Applications, 59, 1425-1432 (2010).

[22] D. A. Molodtsov, Soft set theory-firs tresults, Computers and Mathematics with Applications, 37, 19-31 (1999).

[23] D.Molodtsov,V. Y. Leonov and D. V. Kovkov, Soft sets technique and itsapplication, Nechetkie Sistemy i Myagkie Vychisleniya, 1, 8-39 (2006).
[24] A. Mukherjee and S. B. Chakraborty, On intuitionistic fuzzy soft relations, Bulletin of Kerala Mathematics Association, 5, 35-42 (2008).

[25] D. Pei and D. Miao, From soft sets to information systems, in: X. Hu, Q. Liu, A. Skowron, T. Y. Lin, R. R. Yager, B. Zhang (Eds.), Proceedings of Granular Computing, in: IEEE, 2, 617-621 (2005).

[26] R. L. Newcomb, Topologies which are compact modulo an ideal. Ph.D, Dissertation, Univ. Cal. at Santa Barbara, (1967).

[27] D. V. Rancin, Compactness modul an ideal, Soviet Math. Dokl., 13, 193-197 (1972).

[28] P. Samuels, A topology from a given topology topology and ideals, J. London Math. Soc., (1992).

[29] M. Shabir and M. Naz, On soft topological spaces, Comput. Math. Appl., 61, 1786-1799 (2011).

[30] Z. Xiao, L. Chen, B. Zhong, S. Ye, Recognition for information based on the theory of soft sets, in: J. Chen(Ed.), Proceeding of ICSSSM-05, IEEE, 2, 1104-1106 (2005).

[31] W. Xu, J. Ma, S. Wang and G. Hao, Vague soft sets and their properties, Computers and Mathematics with Applications, 59, 787-794 (2010).

[32] Y. Zou and Z. Xiao, Data analysis approaches of soft sets under incomplete information, Knowledge-Based Systems, 21, 941-945 (2008).

[33] I. Zorlutuna, M. Akdag, W.K. Min and S. Atmaca, Remarks on soft topological spaces, Annals of Fuzzy Mathematics and Informatics, 3, 171-185 (2012).

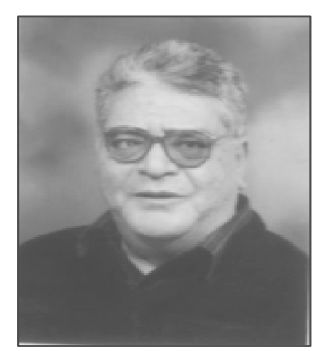

Ali Kandil Saad Ibrahim is a Professor of Mathematics at Helwan University. He received the Ph.D. degree in Topology from the University of Moscow in 1978. His primary research areas are General Topology, Fuzzy Topology, double sets and theory of sets. Dr. Kandil has published over 80 papers in refereed journals and contributed several book chapters in various types of Mathematics textbooks. He is a Fellow of the Egyptian Mathematical Society and Egyptian Physics Mathematical Society. He was the Supervisor of 20 PHD and about 30 MSC students. 


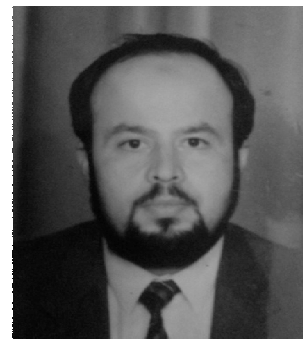

Osama Abd El-Hamid El-Tantawy is a Professor of Mathematics at Zagazig University. He born in 1951. He received the Ph.D. degree in Topology from the University of Zagazig in 1988 . His primary research areas are General Topology, Fuzzy Topology, double sets and theory of sets. Dr. Osama has published over 50 papers in refereed journals. He is a Fellow of the Egyptian Mathematical Society and Egyptian Physics Mathematical Society. He was the Supervisor of 10 PHD and about 17 MSC students.

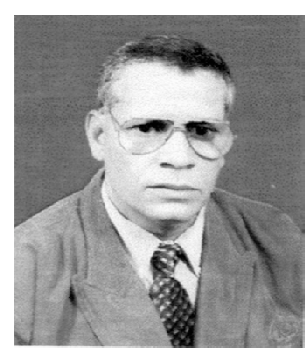

Sobhy Ahmed
Aly El-Sheikh is an
assistance Professor of
pure Mathematics, Ain
Shams University ,Faculty
of Education, Mathematic
Department, Cairo, Egypt. He
born in 1955. He received the

Ph.D. degree in Topology from the University of Zagazig. His primary research areas are General Topology, Fuzzy Topology, double sets and theory of sets. Dr. Sobhy has published over 15 papers in Fuzzy set and system Journal (FSS), Information science Journal (INFS), Journal of fuzzy Mathematics and Egyptian Journal of Mathematical Society. He was the Supervisor of many PHD and MSC Thesis.

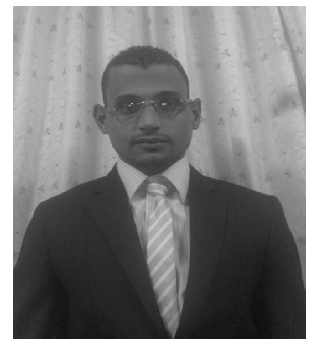

$\begin{array}{cl}\text { Alaa } & \text { Mohamed } \\ \text { Abd El-Latif } & \text { Daby is }\end{array}$

a Ph.D student in pure Mathematics (Topology) Ain Shams University ,Faculty of Education, Mathematic Department, Cairo, Egypt. He was born in 1985. He received the MSC Thesis degree in Topology from Ain Shams University in 2012. His primary research areas are General Topology, Fuzzy Topology, Set theory, Soft set theory and Soft topology. Dr. Alaa has published many papers in refereed journals. 